\title{
In vitro evaluation of the antitumor effect of bismuth lipophilic nanoparticles (BisBAL NPs) on breast cancer cells
}

This article was published in the following Dove Press journal: International Journal of Nanomedicine

\section{Rene Hernandez- Delgadillo,' Claudia María García-Cuéllar, ${ }^{2}$ Yesennia Sánchez-Pérez, ${ }^{2}$ Nayely Pineda-Aguilar, ${ }^{3}$ Marco Antonio Martínez- Martínez,' Eyra Elvyra Rangel-Padilla,' Sergio Eduardo Nakagoshi- Cepeda,' Juan Manuel Solís-Soto,' Rosa Isela Sánchez-Nájera,' María Argelia Akemi Nakagoshi- Cepeda,' Shankararaman Chellam, ${ }^{4}$ Claudio Cabral- Romero' \\ 'Universidad Autónoma de Nuevo León, UANL, Facultad de Odontología, Laboratorio de Biología Molecular, Monterrey, Nuevo León, México; ${ }^{2}$ Subdirección de Investigación Básica, Instituto Nacional de Cancerología, CDMX, México; ${ }^{3}$ Centro de Investigación en Materiales Avanzados, S.C. (CIMAV), Unidad Monterrey, Nuevo León, México; ${ }^{4}$ Texas A\&M University, College Station, TX, USA}

Correspondence: Claudio CabralRomero

Universidad Autónoma de Nuevo León, UANL, Facultad de Odontología, Laboratorio de Biología Molecular, Dr E Aguirre Pequeño and Silaos/n., Mitrascentro, Monterrey, Nuevo León C.P.64460, México

Tel +52 8I8329 4000 ext 3I53

Email claudio.cabralrm@uanl.edu.mx
Aim: The objective of this study was to evaluate the antitumor activity of lipophilic bismuth nanoparticles (BisBAL NPs) on breast cancer cells.

Materials and methods: The effect of varying concentrations of BisBAL NPs was evaluated on human MCF-7 breast cancer cells and on MCF-10A fibrocystic mammary epitheliocytes as noncancer control cells. Cell viability was evaluated with the MTT assay, plasma membrane integrity was analyzed with the calcein AM assay, genotoxicity with the comet assay, and apoptosis with the Annexin V/7-AAD assay.

Results: BisBAL NPs were spherical in shape (average diameter, $28 \mathrm{~nm}$ ) and agglomerated into dense electronic clusters. BisBAL NP induced a dose-dependent growth inhibition. Most importantly, growth inhibition was higher for MCF-7 cells than for MCF-10A cells. At $1 \mu \mathrm{M}$ BisBAL NP, MCF-7 growth inhibition was $51 \%$, while it was $11 \%$ for MCF-10A; at $25 \mu \mathrm{M}$ BisBAL NP, the growth inhibition was $81 \%$ for MCF-7 and $24 \%$ for MCF-10A. With respect to mechanisms of action, a 24-hour exposure of 10 and $100 \mu \mathrm{M}$ BisBAL NP caused loss of cell membrane integrity and fragmentation of tumor cell DNA. BisBAL NPs at $10 \mu \mathrm{M}$ were genotoxic to and caused apoptosis of breast cancer cells.

Conclusion: BisBAL NP-induced growth inhibition is dose dependent, and breast cancer cells are more vulnerable than noncancer breast cells. The mechanism of action of BisBAL NPs may include loss of plasma membrane integrity and a genotoxic effect on the genomic DNA of breast cancer cells.

Keywords: antitumor activity, bismuth nanoparticles, breast cancer, chemotherapy, cytotoxicity

\section{Introduction}

Breast cancer continues to be a major challenge for modern medicine worldwide. ${ }^{1}$ Despite the efforts of the chemical and medicinal industry, breast cancer prevalence is expected to rise. $^{2}$ Conventional treatments, including surgery, chemotherapy, and radiation, not only kill tumor tissue but also healthy tissue. Chemotherapy is the treatment of choice for localized and metastasized cancers. Cancer tissue distinguishes itself from normal tissue in the following aspects: sustained proliferative signaling, evasion of growth suppressors, resistance to cell death, replicative immortality, induction of angiogenesis, invasion, and metastasis. ${ }^{3}$ Traditional chemotherapy not only kills the tumor but also damages normal healthy tissue. There is an ongoing effort for developing selective antitumor agents that attack tumor tissue with a higher efficacy while sparing healthy tissue and diminishing side effects. Several metallic nanostructures, including silver, gold, and selenium nanoparticles (NPs), have been analyzed 
to inhibit the growth of breast cancer cells. ${ }^{4-6}$ Nonmetallic NPs have been used as a vehicle for "specific drug delivery" at the tumor site. ${ }^{7}$

Bismuth is the heaviest member of the pnictogen group and is often referred to as a "green" element. ${ }^{8}$ In medicine, bismuth subsalicylate (ie, Pepto-Bismol) is used to treat diarrhea, indigestion, and nausea. Although bismuth has no direct application against cancer cells, several bismuth-based compounds have potent anticancer activity, eg, thiosemicarbazone, hydrazone, and dithiocarbamate. ${ }^{9-12}$ Hydrazone derivatives are well-known to possess antimicrobial activity, but the Bi(III) hydrazine complex proved to be the most active form against several cancer cell lines (Jurkat, HL60, MCF-7, and HCT-116). ${ }^{13,14}$ A novel bismuth-sulfapyridine complex that inhibits the growth of leukemia cells (K562) ${ }^{15}$ and heterocyclic organobismuth(III) compounds, which were investigated as antimicrobial agents, turned out to have significant anticancer activity against several human cancer cell lines. ${ }^{16}$ Interestingly, the aforementioned bismuth compounds have both antimicrobial and anticancer activities. Recently, our group described the excellent antimicrobial properties of lipophilic bismuth NPs (BisBAL NPs) with a minimal inhibitory concentration of 5-10 $\mu \mathrm{M}$ against several oral pathogens. ${ }^{17}$ However, the potential anticancer activity of bismuth NPs has not been extensively explored. A recent report describes how tumor-targeted bismuth NPs enhanced $\mathrm{X}$-ray radiation therapy against breast cancer cells. ${ }^{18}$

In this work, we report for the first time that BisBAL NPs selectively inhibit the growth of breast cancer cells in a dose-dependent way. BisBAL NPs seem a promising innovative alternative in cancer chemotherapy that deserves to be evaluated in other cancer cell lines and animal models.

\section{Materials and methods}

\section{Synthesis and characterization of BisBAL NPs}

To synthesize BisBAL NPs, the colloidal method according to Badireddy et al was used. ${ }^{17}$ Briefly, $0.485 \mathrm{~g}$ of Bi( $\left(\mathrm{NO}_{3}\right)_{3} \cdot 5 \mathrm{H}_{2} \mathrm{O}$ was dissolved in $20 \mathrm{~mL}$ propylene glycol, heated to $80^{\circ} \mathrm{C}$, and agitated for 2 hours to obtain a $50 \mathrm{mM} \mathrm{Bi}^{3+}$ solution. A 2:1 molar ratio of $\mathrm{Bi}^{3+}$ (Bis) to 2,3-dimercapto-1-propanol (BAL) was prepared by adding $25 \mu \mathrm{L} 10 \mathrm{M}$ BAL to 10 $\mathrm{mL} 50 \mathrm{mM} \mathrm{Bi}^{3+}$ solution. A stock suspension of $25 \mathrm{mM}$ BisBAL NPs was prepared by adding 0.85 volumes (V) of ice-cold ultrapure water and $0.15 \mathrm{~V}$ of freshly prepared ice-cold $75 \mathrm{mM} \mathrm{NaBH}_{4}$ to $5 \mathrm{~mL} 50 \mathrm{mM}$ BisBAL NP under continuous vigorous mixing; the pink-colored BisBAL solution instantly transformed to a black-colored suspension of
BisBAL NPs. The distribution of BisBAL NP's size and shape was analyzed with scanning electron microscopy (SEM; Nova NanoSEM 200, FEI Company, Eindhoven, the Netherlands; $15 \mathrm{kV}$ ). The presence of bismuth was corroborated by energy-dispersive X-ray spectroscopy (EDS) SEM. For structural analysis, X-ray diffractometry patterns were obtained from water-washed (three centrifugation cycles, 16,100×g, 30 seconds), air-dried BisBAL NPs (deposited several times on a glass slide overnight) using an X-ray diffractometer (Panalytical X'Pert PRO MRD) equipped with $\mathrm{Cu} \mathrm{K} \alpha$ as an X-ray source $(\lambda=1.541874 \AA)$. The ultraviolet (UV)-visible spectrum of the BisBAL NP suspension was recorded using a spectrophotometer (SpectraMax Plus384 Absorbance Microplate Reader; Molecular Devices, LLC, Sunnyvale, CA, USA).

\section{Cell culture}

The human breast carcinoma cell line MCF-7 was obtained from the American Type Culture Collection (ATCC HTB-22; ATCC, Rockville, MD, USA). A fibrocystic mammary gland epithelial cell line, MCF-10A (ATCC CRL-10317; ATCC) was used as a nontumor control. MCF-7 cells were cultivated in DMEM/Ham's F12 (DMEM/F12) supplemented with 10\% FBS (Gibco-Invitrogen, Carlsbad, CA, USA) and $100 \mathrm{U} / \mathrm{mL}$ penicillin, $100 \mu \mathrm{g} / \mathrm{mL}$ streptomycin, and $0.25 \mu \mathrm{g} / \mathrm{mL}$ amphotericin B (Sigma-Aldrich Corporation, St Louis, MO, USA) in cell culture flasks (Corning Inc., Corning, NY, USA) at $37^{\circ} \mathrm{C}$ in a humidified atmosphere with $5 \% \mathrm{CO}_{2}{ }^{19} \mathrm{MCF}-10 \mathrm{~A}$ cells were cultivated in mammary epithelial cell growth medium supplemented with $100 \mathrm{ng} / \mathrm{mL}$ cholera toxin in cell culture flasks (Corning Inc.) at $37^{\circ} \mathrm{C}$ and $5 \% \mathrm{CO}_{2}$. Cells of a confluent monolayer were harvested by scraping, washed three times with $10 \mathrm{mM}$ PBS, pH 7.4 (PBS), and counted with a hemocytometer.

\section{MTT cell viability assay}

The effect of BisBAL NPs on MCF-7 and MCF-10A cell number was evaluated using the MTT cell viability assay (Biotium, Hayward, CA, USA). ${ }^{20}$ Then, $1 \times 10^{5}$ cells were incubated with $0,0.125,1,10,25,50$, and $100 \mu \mathrm{M}$ of BisBAL NPs or 1,000 $\mu \mathrm{M}$ doxorubicin (Doxolem, Teva Lab, Madrid, Spain). The treatment was terminated by washing the cells with PBS. Next, cells were incubated with MTT $(10 \mu \mathrm{L} /$ well) at $37^{\circ} \mathrm{C}$ and $5 \% \mathrm{CO}_{2}$ for 2 hours in the dark according to the manufacturer's instructions. Next, the medium was removed, and $100 \mu \mathrm{L}$ of dimethylsulfoxide (DMSO) was added to dissolve the reduced MTT formazan product. To quantify the reduced MTT, the absorbance at $570 \mathrm{~nm}\left(\mathrm{~A}_{570}\right)$ 
was measured by microplate absorbance reader (BioTek, Winooski, VT, USA); DMSO served as a blank. The assay was performed in triplicate.

\section{Cell membrane permeability by fluorescence microscopy}

Calcein AM is an excellent tool to study cell membrane integrity. Calcein AM is membrane-permeant and can be introduced into cells via incubation. Once inside the cells, nonfluorescent calcein AM is hydrolyzed by endogenous esterase into a highly charged green fluorescent molecule that is retained in the cytoplasm of living cells. Therefore, intracellular calcein AM in living cells was interpreted as a signal of intact cell membrane. This assay was used to analyze the cell membrane permeability of MCF-7 cells after a 24-hour exposure to $0,1,10$, and $100 \mu \mathrm{M}$ BisBAL $\mathrm{NP}$; $0 \mu \mathrm{M}$ BisBAL NP, ie, cells exposed to pure culture medium, served as a growth control. After incubation, cells were washed three times with PBS and stained with $2 \mu \mathrm{M}$ calcein AM (Biotium) for 30 minutes at $37^{\circ} \mathrm{C} .{ }^{21}$ Nuclei of MCF-7 cells were stained with $1 \mu \mathrm{g} / \mathrm{mL}$ DAPI (Abcam Inc, Cambridge, UK). ${ }^{22}$ Next, cells were washed again with PBS and air-dried in the dark. Cell morphology was observed with fluorescein isothiocyanate (FITC) and DAPI filters at 485 and $358 \mathrm{~nm}$, respectively (Thornwood, NY, USA). A degraded or amorphous nucleus was interpreted as a potential genotoxic effect.

\section{Genotoxicity by comet assay and fluorescence microscopy}

To determine the possible genotoxic effect of BisBAL NPs, MCF-7 cells were incubated overnight at $37^{\circ} \mathrm{C} / 5 \% \mathrm{CO}_{2}$ with 1 or $10 \mu \mathrm{M}$ BisBAL NP, $100 \mu \mathrm{M}$ etoposide (Sigma) as a positive control, or culture medium as a growth control. After incubation, cell damage was determined with the OxiSelect ${ }^{\mathrm{TM}}$ Comet Assay Kit (Cell Biolabs, Inc, San Diego, CA, USA) according to the manufacturer's instructions. ${ }^{23}$ Briefly, cells were harvested by scraping and centrifugation (700 $\times g, 2$ minutes) and washed with PBS. Cell suspensions were mixed with liquefied Comet Agarose at a 1:10 ratio (v/v) and pipetted on an OxiSelect Comet Slide $(75 \mu \mathrm{L} /$ well $)$. After a 15 -minute embedding step $\left(4^{\circ} \mathrm{C}\right.$, dark, horizontal position), cells were lysed ( $25 \mathrm{~mL}$ lysis buffer/slide, 30-minute incubation, $4^{\circ} \mathrm{C}$, dark, horizontal position) and treated with an alkaline solution $\left(25 \mathrm{~mL} /\right.$ slide, 30 minutes, $4^{\circ} \mathrm{C}$, dark) to relax and denature the DNA. Finally, the samples are electrophoresed in a horizontal chamber $(300 \mathrm{~mA}$ for 30 minutes) to separate intact DNA from damaged fragments.
Next, samples are washed with sterile MilliQ water, treated with 70\% cold ethanol for 5 minutes, air-dried, and stained with the DNA dye DAPI (100 $\mu \mathrm{L} /$ well), and viewed with an epifluorescence microscope using a DAPI filter (Thornwood, NY, USA).

\section{Apoptosis evaluation by phosphatidylserine-expressing plasma membrane by fluorescence microscopy}

In healthy cells, phosphatidylserine is located in the inner leaflet of the plasma membrane. Apoptotic cells, on the contrary translocate phosphatidylserine to the outer leaflet of the plasma membrane to be recognized by phagocytes. In order to analyze whether BisBAL NPs promote apoptosis among cancer cell lines, the $\mathrm{CF}^{\circledR} 488 \mathrm{~A}$ Annexin $\mathrm{V}$ and 7-AAD Apoptosis Kit (Biotium) was used according to the manufacturer's instructions. Briefly, cells $\left(1 \times 10^{4} /\right.$ well in a 96-well plate) were cultured for 24 hours at $37^{\circ} \mathrm{C} / 5 \%$ $\mathrm{CO}_{2}$ in DMEM/F12 and exposed for 3 hours to 1 or $10 \mu \mathrm{M}$ BisBAL NP, $100 \mu \mathrm{M}$ etoposide (Sigma) as positive control of cell death, or pure culture medium as a growth control. Next, cells harvested by scraping were washed three times with PBS, resuspended in $100 \mu \mathrm{L} 1 \times$ annexin binding buffer, and incubated (30 minutes, on ice, dark) with $5 \mu \mathrm{L}$ CF488A-Annexin $\mathrm{V}$ and $2 \mu \mathrm{L}$ of 7-AAD working solution. Next, cells were observed with a fluorescence microscope using FITC and CY5 filters (Thornwood, NY, USA).

\section{Statistical analysis}

A multiple comparison 2-way ANOVA with Tukey's correction was used to compare all groups. For all statistical tests, a significance level of $\alpha=0.05$ was considered.

\section{Results}

\section{Characterization of BisBAL NPs}

The obtained BisBAL NPs were spherical in shape with an average diameter of $28 \mathrm{~nm}$. The NPs were composed of rhombohedral crystallites $(\approx 18 \mathrm{~nm})$ with dithiols as lipophilic surface groups and the lattice spacing of individual crystallite was $0.325 \mathrm{~nm}$. The specific presence of bismuth was confirmed by EDS-SEM. The BisBAL NPs formed electrodense clusters (Figure 1). UV-visible absorbance revealed that BisBAL NPs had a higher $(\approx 70 \%)$ affinity for 1-octanol rather than for water, consistent with a lipophilic property of the dithiols bound to NP surface. BisBAL NPs resulted to be stable for at least 2 months at $4^{\circ} \mathrm{C}$ and room temperature. 
A

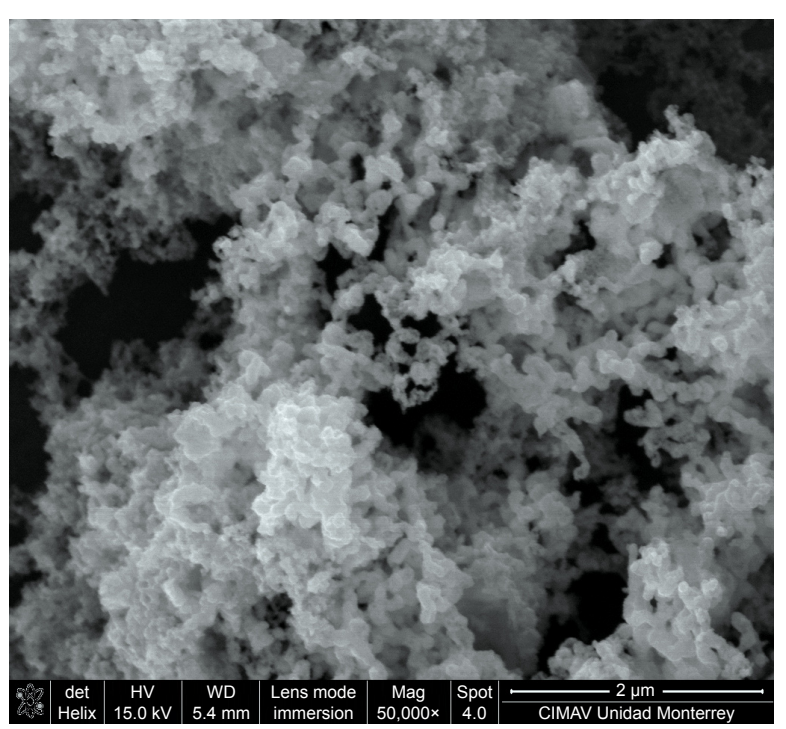

C

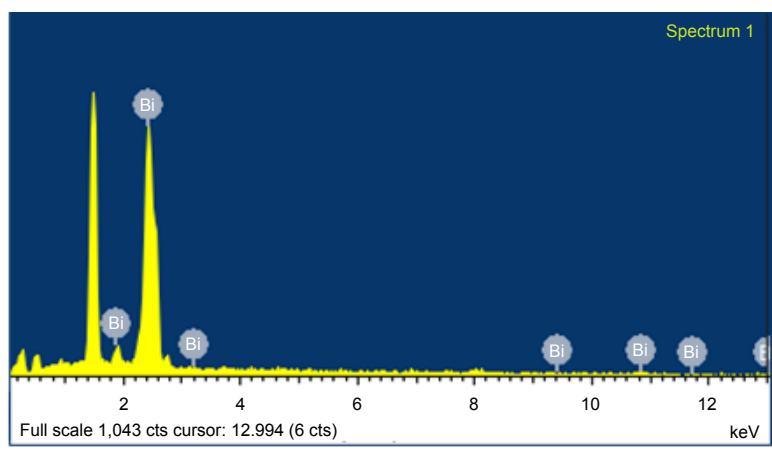

B

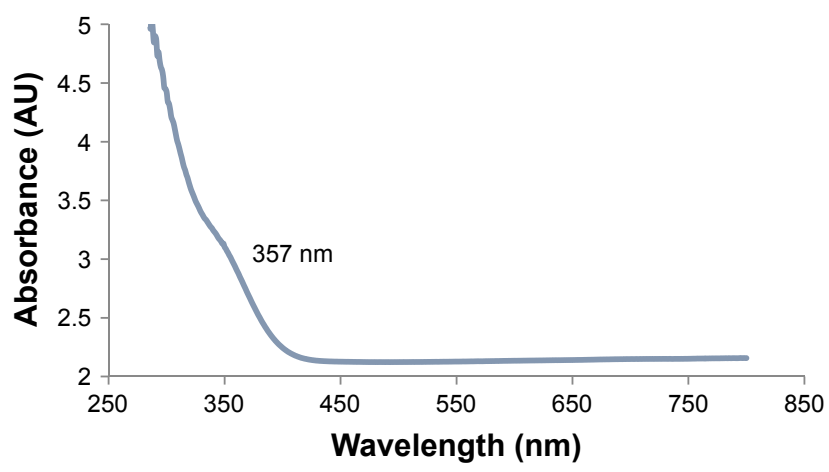

D

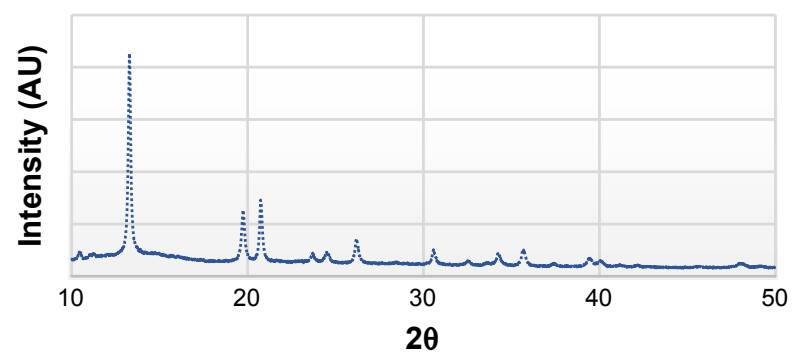

Figure I Characterization of lipophilic bismuth nanoparticles (BisBAL NPs).

Notes: (A) Shape, size, and shape-and-size distribution of BisBAL NPs were obtained by scanning electron microscopy. (C) The presence of bismuth was confirmed by the emission lines characteristic of this element, as can be seen in the spectrum. The signal at $1.486 \mathrm{keV}$ is due to the aluminum substrate where the sample was placed. (B) and (D) The UV-visible absorbance and the X-ray diffractometry pattern of BisBAL NPs.

\section{Antitumor activity}

MCF-7 tumor cells that had been exposed to $1 \mu \mathrm{M}$ BisBAL NP grew 50\% less than MCF-7 that had not been exposed to BisBAL NPs (Figure 2). Unlike tumor cells, the growth of control cells decreased only $10.5 \%$ (Figure 2 ). When exposed to a higher dose ( $25 \mu \mathrm{M}$ BisBAL NP), tumor cell growth was $80.4 \%$ less and control cells grew 26\% less than nonexposed cells. Exposure to 1,000 $\mu \mathrm{M}$ doxorubicin diminished MCF-7 cell growth by $98 \%$. Thus, BisBAL NPs selectively inhibit tumor cell growth in a dose-dependent way.

\section{Cell membrane permeability alteration after exposure to BisBAL NPs}

After a 24-hour exposure to $1 \mu \mathrm{M}$ BisBAL NP, MCF-7 morphology changed; cells rounded up and released calcein AM, indicating loss of cell membrane integrity (Figure 3 ). This effect was more pronounced after exposure to $10 \mu \mathrm{M}$ BisBAL NP, whereas after exposure to $100 \mu \mathrm{M}$ BisBAL NP only cell debris was observed. These results are consistent with the MTT cell viability assay results, where $95 \%$ of tumor cells died at $100 \mu \mathrm{M}$ BisBAL NP.

\section{Genotoxic assays}

Possible damage to genomic DNA of breast cancer cells was analyzed with the comet assay and fluorescence microscopy, in which $100 \mu \mathrm{M}$ etoposide served as a positive control of DNA break induction. As of $10 \mu \mathrm{M}$ BisBAL NP, "comets" identical to the ones in the etoposide control were observed (Figure 4), suggesting that $10 \mu \mathrm{M}$ BisBAL NP promotes DNA strand breaks. Interestingly, $1 \mu \mathrm{M}$ BisBAL NP did not alter genomic DNA.

\section{Apoptosis assays}

Programmed cell death is a response to different kinds of stimuli. To analyze if BisBAL NPs could induce apoptosis among MCF-7 cells, the Annexin V, 7-AAD assay, 


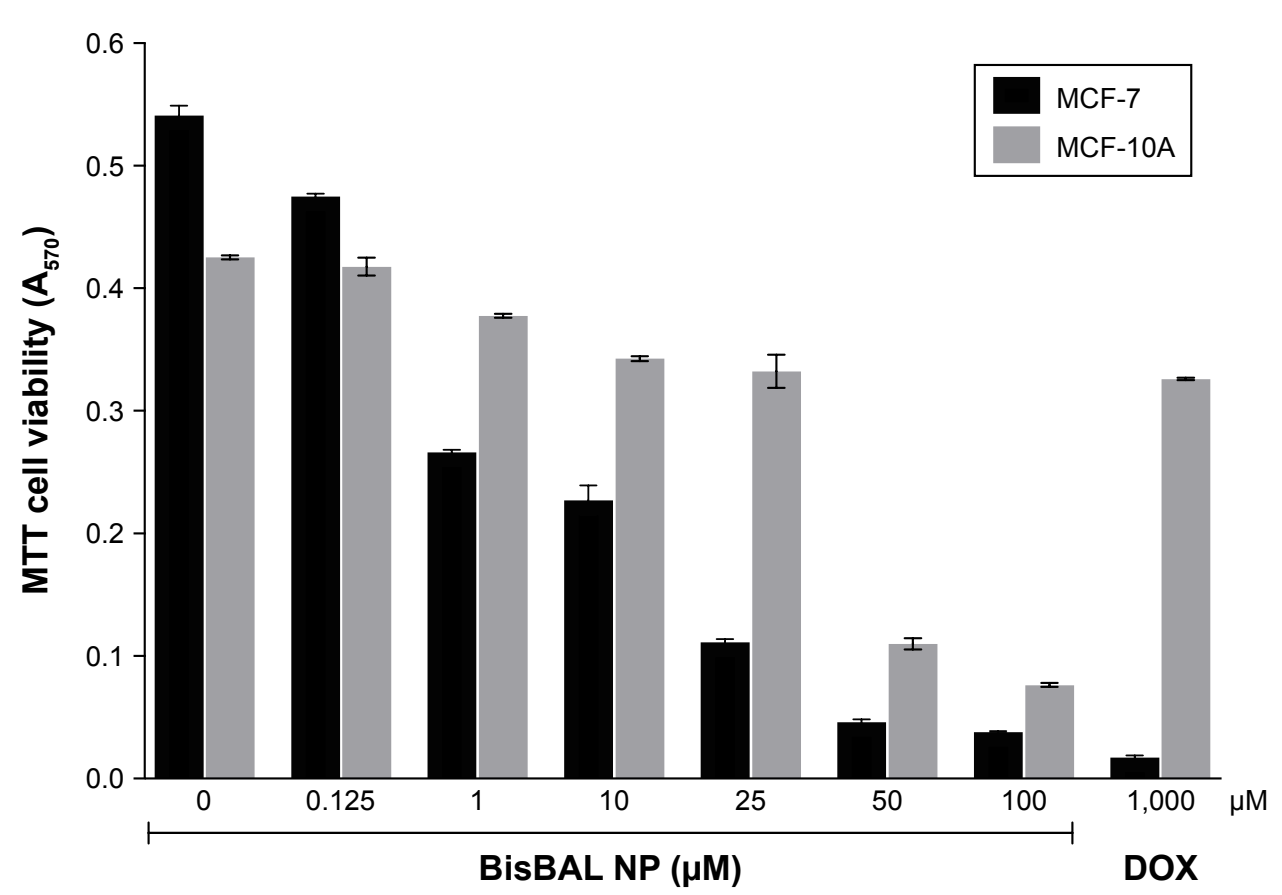

Figure 2 Antitumor activity of BisBAL NPs on the human breast cancer cell line MCF-7.

Notes: Cell viability of MCF-7 (human breast cancer cell line) and MCF-IOA (nontumor human breast cancer cell line) was determined by the MTT cell viability assay at $\mathrm{A}_{570}$ after a 24-hour exposure to $0,0.125,1,10,25,50$, and $100 \mu \mathrm{M}$ BisBAL NP and I,000 $\mu$ M DOX (positive control of cytotoxicity). After a multiple comparison 2-way ANOVA with Tukey's correction, all samples were significantly different $(P<0.000$ I), except for: I) 50 vs I00 $\mu M$ BisBAL NP exposure for MCF-7 cells, 2$) 0$ vs $0.125 \mu M$ BisBAL NP exposure for MCF-IOA cells, and 3) among 10 and $25 \mu \mathrm{M}$ BisBAL NP, and I,000 $\mu \mathrm{M}$ DOX exposure for MCF-I0A cells. Bars indicate mean \pm SD ( $\mathrm{n}=4$ for MCF-7 and $n=3$ for MCF-IOA).

Abbreviations: DOX, doxorubicin; BisBAL NPs, lipophilic bismuth nanoparticles.

and fluorescence microscopy were used. BisBAL NPs at $1-10 \mu \mathrm{M}$ yielded results that were very similar to the positive control of $100 \mu \mathrm{M}$ etoposide (Figure 5). In contrast, no signals of programmed cell death were observed in the growth control. Altogether these results suggest that low concentrations of bismuth NPs promote apoptosis among breast cancer cells.

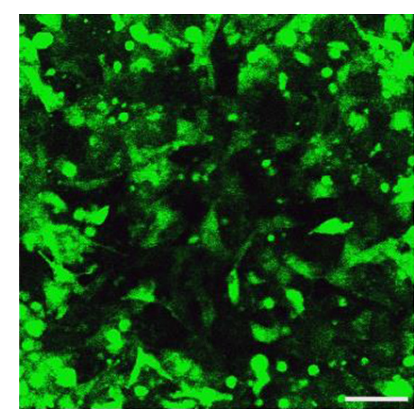

MCF-7

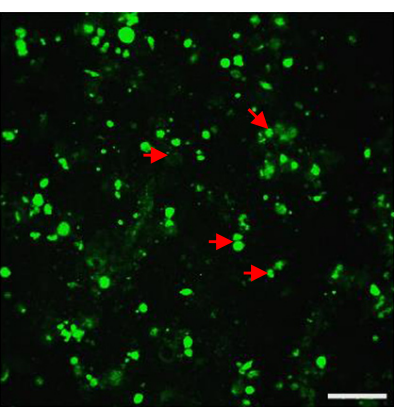

1

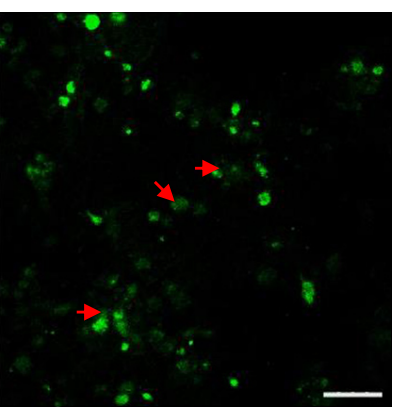

10

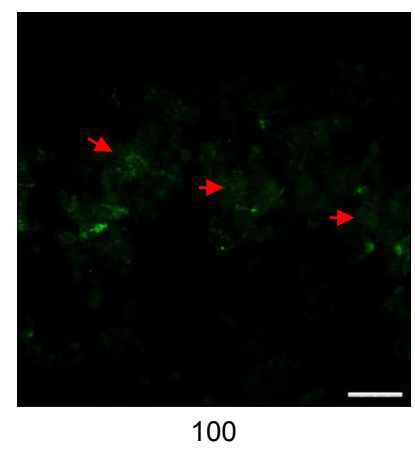

100

BisBAL NPs $(\mu \mathrm{M})$

Figure 3 Effect of BisBAL NPs on cell membrane integrity of breast cancer cells evaluated with the calcein AM assay.

Notes: Fluorescent $(485 \mathrm{~nm})$ calcein AM distribution (within or outside MCF-7 cells) after a 24-hour exposure to I, 10 , or I00 $\mu$ M BisBAL NP for 24 hours. Red arrow indicates released calcein AM. Bar, $5 \mu \mathrm{m}$.

Abbreviation: BisBAL NPs, lipophilic bismuth nanoparticles. 


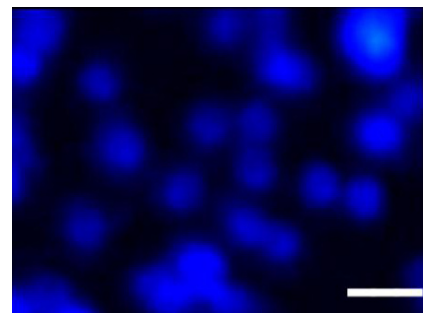

Growing control

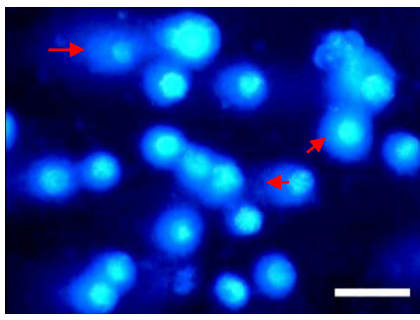

$100 \mu \mathrm{M}$ etoposide

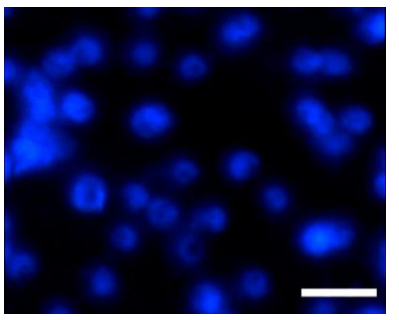

1

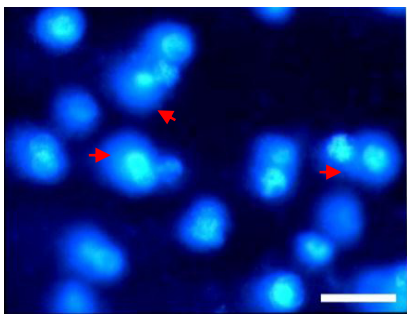

10

BisBAL $(\mu \mathrm{M})$

Figure 4 Genotoxic effect of BisBAL NPs on MCF-7 breast cancer cells.

Notes: MCF-7 cells were incubated for 24 hours with culture medium as a growth control of intact cells, $100 \mu$ M etoposide (positive control of genotoxic effect), and I or $10 \mu \mathrm{M}$ BisBAL NPs. DNA damage was evaluated with the OxiSelect ${ }^{\mathrm{TM}}$ Comet Assay Kit, and DAPI-stained DNA was observed with an epifluorescence microscope with DAPI filter. Red arrow indicates the stellar morphology. Bar, $5 \mu \mathrm{m}$.

Abbreviation: BisBAL NPs, lipophilic bismuth nanoparticles.

cell death subroutines that includes extrinsic and intrinsic apoptosis, regulated necrosis, autophagic cell death, and mitotic catastrophe. ${ }^{24}$ For each subroutine, measurable biochemical features have been defined.
Nanomedicine is a new discipline that offers the possibility to develop "smart drugs" that allows controlled target-specific, drug delivery controlled with less adverse effects than nontargetted drugs..$^{25,26}$ Nanocomposites are
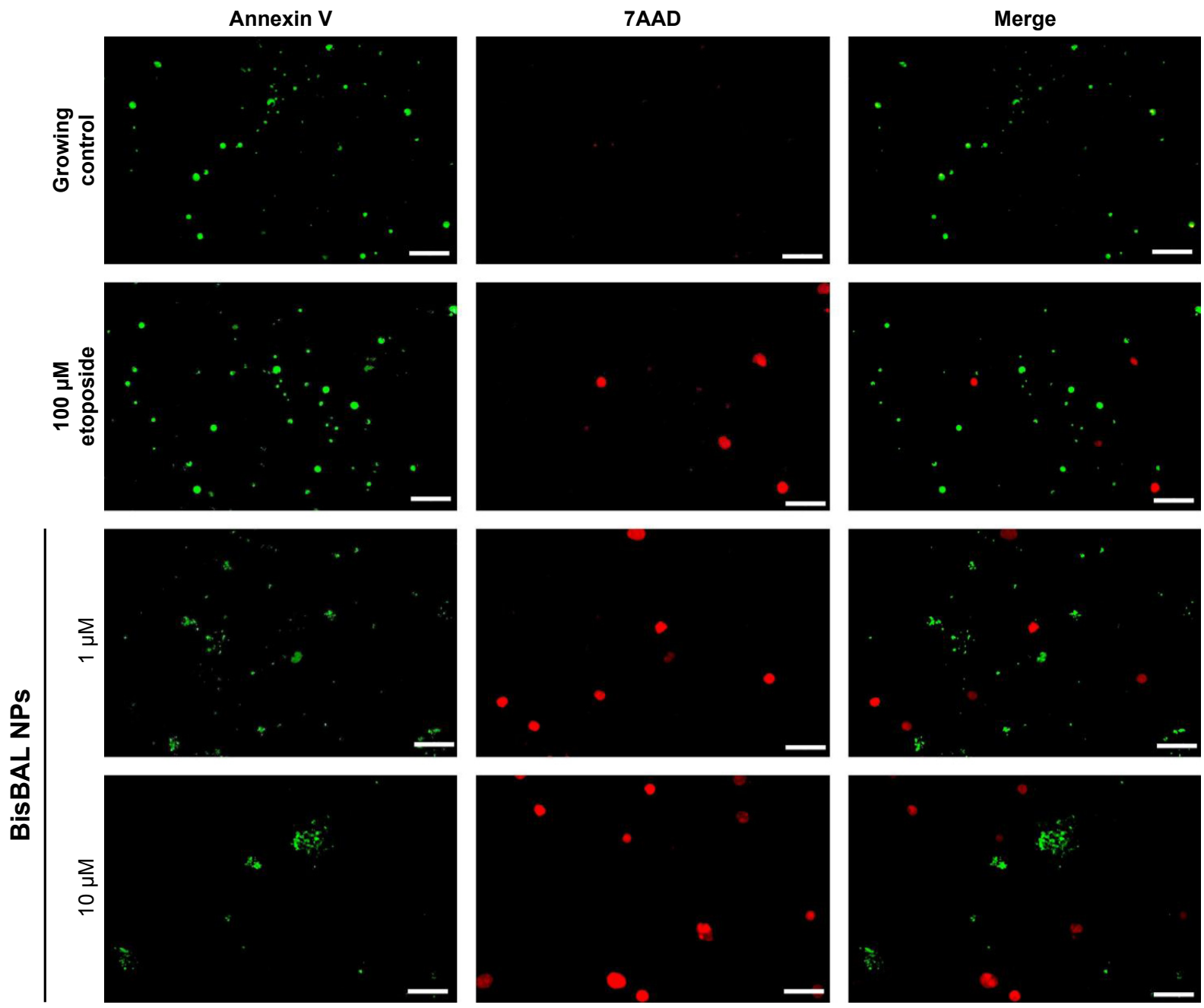

Figure 5 Detection of apoptosis in human breast cancer cells by BisBAL NPs.

Notes: Apoptosis induction in MCF-7 cells by BisBAL NPs was detected with the CF ${ }^{\circledR} 488 \mathrm{~A}$ Annexin $\mathrm{V}$ and 7-AAD apoptosis assay. Etoposide at I00 $\mu$ M was used as a positive control of an apoptotic agent, whereas pure culture medium was used as a negative control; I and $10 \mu M$ BisBAL NPs were analyzed on MCF-7 cells. After treatments, cell cultures were observed with a fluorescence microscope using fluorescein isothiocyanate (green) and CY5 (red) filters. Bar, $5 \mu$ m. 
applied in tissue engineering and imaging for diagnostic and therapeutic purposes, including cancer diagnosis and treatment. Different metal NPs (silver, gold, platinum, magnesium, copper, etc) are applied in the treatment of cancer. The NP may have direct or indirect antitumor activity. In direct antitumor activity, the NP itself may inhibit growth or survival of tumor cells, whereas the induction of heat would be an example of indirect antitumor activity of NPs (photothermal therapy). ${ }^{27}$ Several groups have reported anticancer activity of different types of metal NPs, such as silver, gold, platinum, magnesium, and copper NPs. ${ }^{18,28,29}$ Unfortunately, aforementioned metal NPs are toxic for human cells, which restricts their application in the clinic. ${ }^{30,31}$ As opposed to aforementioned metal and others such as aluminum and antimony, bismuth is considered to be nontoxic. ${ }^{32}$ In medicine, bismuth subsalicylate is used to treat diarrhea without cytotoxic reports. ${ }^{33} \mathrm{We}$ previously reported the excellent bactericidal, antifungal, and antibiofilm activities of bismuth NPs. ${ }^{17}$ Furthermore, BisBAL NPs are not toxic for human epithelial and blood cells. . $^{32,34}$

In this work, we demonstrated the effectiveness of bismuth NPs to inhibit the growth of human tumor cells selectively because $1 \mu \mathrm{M}$ of BisBAL NPs decreased the growth of tumor cell growth with $51 \%$, while the growth of control cells was unaltered. Employing in vitro assays Wang et al (2012) described that cuprous oxide NPs selectively induce apoptosis of tumor cells. ${ }^{35}$ On the contrary, Deng et al reported the indirect antitumor activity of bismuth NPs. They used bismuth NPs as radiosensitizers to enhance X-ray radiation therapy and achieved a significant tumor reduction. ${ }^{18}$ In our study, the antitumor effect of BisBAL NPs was not only direct and selective but also dose-dependent. In our in vitro set up, the therapeutic range was $1-25 \mu \mathrm{M}$ BisBAL NP. In these range, antitumor activity was selective as it hardly had adverse effects on healthy cells. The anticancer activity of silver NPs on the human colon adenocarcinoma cell line HT-29 is also dose-dependent. ${ }^{36}$ Apart from having anticancer activity, NPs in the form of polymeric carbon nanotubes have been proposed to serve as carriers of chemotherapeutic agents by facilitating an increased permeability and retention within the tumor. ${ }^{37,38}$

The anticancer potential of NPs was screened for against the MCF-7 cancer cell line and the MCF-10A normal cell line as a control with the calcein AM assay. The anticancer results were comparable with the anticancer drug (cisplatin), but without damaging the control cell line. ${ }^{39}$

Based on the calcein AM assay, we suggest that the possible anticancer action mechanism of BisBAL NPs is altering the cell membrane integrity of tumor cells. After a 24-hour exposure to $100 \mu \mathrm{M}$ BisBAL NP, MCF-7 cells look completely destroyed; an effect not seen after exposure to $10 \mu \mathrm{M}$ BisBAL NP. Similar results have been reported for silver NPs that had been synthetized with a "green method." Copper ferrite NPs also decreased cell viability and caused membrane damage in a dose-dependent way in MCF-7 breast cancer cells. ${ }^{40}$ We hypothesize that the water solubility and lipophilicity of bismuth is enhanced when bismuth ions are complexed with small lipophilic molecules such as dimercaptopropanol. It is highly probable that the lipophilic character of BisBAL NPs is responsible for an increased affinity to cell membranes of breast cancer cells compared with nonlipophilic bismuth presentations. The cellular uptake of several anticancer composites has been improved after increasing the lipophilicity of the composites. ${ }^{41,42}$ Another strategy to improve drug delivery while protecting the drug is the encapsulation of the compound in liposomes. ${ }^{41}$

The genotoxicity experiments revealed that the genomic DNA of exposed tumor cells to $1-10 \mu \mathrm{M}$ BisBAL NP was significantly more damaged than the DNA of nonexposed tumor cells. The genotoxicity of silver, gold, and platinum NPs on different cell lines is well-known. ${ }^{43}$ Using the same comet assay as we did, Lebedová et al recently reported DNA damage induced by silver NPs. The effect was independent of NP size. ${ }^{43}$ On the contrary, the DNA damage induced by gold and platinum NPs was NP size-dependent. ${ }^{43}$ Liu et al described the cytotoxic and genotoxic effects of a 24-hour exposure of liver cells to cobalt NPs. Interestingly, cobalt ions were less cytotoxic than cobalt nanostructures. ${ }^{44}$

Our results indicate that low concentrations of BisBAL NPs induced apoptosis among MCF-7 cells. Likewise, Banu et al reported that silver and gold NPs promote apoptosis and regulate $\mathrm{p} 53$ and $\mathrm{Bcl}-2$ expression in human breast adenocarcinoma cells. ${ }^{45}$ Azizi et al described a cytotoxic effect, via apoptosis, of albumin-coated copper NPs on MCF-7 cells, ${ }^{46}$ suggesting that they could be a new chemotherapeutic agent against invasive breast cancer. Our results with BisBAL NPs suggest that concentrations up to $10 \mu \mathrm{M}$ kill tumor cells selectively, probably by disrupting the cell membrane before causing damage to genomic DNA at higher concentrations. Altogether, our results suggest that BisBAL NPs could alter the plasma cell membrane during their entry and diminish its permeability. Once internalized, BisBAL NPs damage the genomic DNA by direct interaction.

In conclusion, BisBAL NPs inhibit human tumor cell growth selectively and in dose-dependent way. The possible action mechanisms of BisBAL NPs may be altering the membrane permeability of breast cancer cells and causing damage to the genomic DNA. 


\section{Acknowledgments}

CC-R thanks the CONACYT for grant 183825. All authors are grateful to Dr Ismael Malagón Santiago for his help in the statistical analysis and Irene Meester, $\mathrm{PhD}$, for critically reviewing and improving the manuscript.

\section{Disclosure}

The authors report no conflicts of interest in this work.

\section{References}

1. Eccles SA, Aboagye EO, Ali S, et al. Critical research gaps and translational priorities for the successful prevention and treatment of breast cancer. Breast Cancer Res. 2013;15(5):R92.

2. Rahib L, Smith BD, Aizenberg R, Rosenzweig AB, Fleshman JM, Matrisian LM. Projecting cancer incidence and deaths to 2030: the unexpected burden of thyroid, liver, and pancreas cancers in the United States. Cancer Res. 2014;74(11):2913-2921.

3. Hanahan D, Weinberg RA. Hallmarks of cancer: the next generation. Cell. 2011;144(5):646-674.

4. Oves M, Aslam M, Rauf MA, et al. Antimicrobial and anticancer activities of silver nanoparticles synthesized from the root hair extract of Phoenix dactylifera. Mater Sci Eng C Mater Biol Appl. 2018;89: 429-443.

5. Fathy MM, Mohamed FS, Elbialy N, Elshemey WM. Multifunctional chitosan-capped gold nanoparticles for enhanced cancer chemoradiotherapy: an in vitro study. Phys Med. 2018;48:76-83.

6. Shahverdi AR, Shahverdi F, Faghfuri E, et al. Characterization of folic acid surface-coated selenium nanoparticles and corresponding in vitro and in vivo effects against breast cancer. Arch Med Res. 2018;49(1): $10-17$.

7. Liu S, Ono RJ, Yang C, et al. Dual pH-responsive shell-cleavable polycarbonate micellar nanoparticles for in vivo anticancer drug delivery. ACS Appl Mater Interfaces. 2018;10(23):19355-19364.

8. Mohan R. Green bismuth. Nat Chem. 2010;2(4):336.

9. Li M, Lu Y, Yang M, Li Y, Zhang L, Xie S. One dodecahedral bismuth(III) complex derived from 2-acetylpyridine N(4)-pyridylthiosemicarbazone: synthesis, crystal structure and biological evaluation. Dalton Trans. 2012;41(41):12882-12887.

10. Li MX, Zhang LZ, Yang M, Niu JY, Zhou J. Synthesis, crystal structures, in vitro biological evaluation of zinc(II) and bismuth(III) complexes of 2-acetylpyrazine N(4)-phenylthiosemicarbazone. Bioorg Med Chem Lett. 2012;22(7):2418-2423.

11. Li YK, Yang M, Li MX, Yu H, Wu HC, Xie SQ. Synthesis, crystal structure and biological evaluation of a main group seven-coordinated bismuth(III) complex with 2-acetylpyridine N4-phenylthiosemicarbazone. Bioorg Med Chem Lett. 2013;23(8):2288-2292.

12. Zhang N, Tai Y, Li M, Ma P, Zhao J, Niu J. Main group bismuth(III), gallium(III) and diorganotin(IV) complexes derived from bis(2acetylpyrazine)thiocarbonohydrazone: synthesis, crystal structures and biological evaluation. Dalton Trans. 2014;43(13):5182-5189.

13. Ferraz KS, Silva NF, da Silva JG, et al. Investigation on the pharmacological profile of 2,6-diacetylpyridine bis(benzoylhydrazone) derivatives and their antimony(III) and bismuth(III) complexes. Eur J Med Chem. 2012;53:98-106.

14. Keogan DM, Griffith DM. Current and potential applications of bismuth-based drugs. Molecules. 2014;19(9):15258-15297.

15. Marzano IM, Franco MS, Silva PP, et al. Crystal structure, antibacterial and cytotoxic activities of a new complex of bismuth(III) with sulfapyridine. Molecules. 2013;18(2):1464-1476.

16. Iuchi K, Hatano Y, Yagura T. Heterocyclic organobismuth(III) induces apoptosis of human promyelocytic leukemic cells through activation of caspases and mitochondrial perturbation. Biochem Pharmacol. 2008; 76(8):974-986.
17. Badireddy AR, Hernandez-Delgadillo R, Sánchez-Nájera RI, Chellam S, Cabral-Romero C. Synthesis and characterization of lipophilic bismuth dimercaptopropanol nanoparticles and their effects on oral microorganisms growth and biofilm formation. J Nanopart Res. 2014;16(6):2456.

18. Deng J, Xu S, Hu W, Xun X, Zheng L, Su M. Tumor targeted, stealthy and degradable bismuth nanoparticles for enhanced X-ray radiation therapy of breast cancer. Biomaterials. 2018;154:24-33.

19. Paramita P, Wardhani BW, Wanandi SI, Louisa M. Curcumin for the prevention of epithelial-mesenchymal transition in endoxifentreated MCF-7 breast cancer cel. Asian Pac J Cancer Prev. 2018;19(5): $1243-1249$.

20. Liu Y, Peterson DA, Kimura H, Schubert D. Mechanism of cellular 3-(4,5-dimethylthiazol-2-yl)-2,5-diphenyltetrazolium bromide (MTT) reduction. J Neurochem. 1997;69(2):581-593.

21. Bozyczko-Coyne D, Mckenna BW, Connors TJ, Neff NT. A rapid fluorometric assay to measure neuronal survival in vitro. J Neurosci Methods. 1993;50(2):205-216.

22. Kubista M, Akerman B, Nordén B. Characterization of interaction between DNA and 4',6-diamidino-2-phenylindole by optical spectroscopy. Biochemistry. 1987;26(14):4545-4553.

23. Ostling O, Johanson KJ. Microelectrophoretic study of radiation-induced DNA damages in individual mammalian cells. Biochem Biophys Res Commun. 1984;123(1):291-298.

24. Galluzzi L, Vitale I, Abrams JM, et al. Molecular definitions of cell death subroutines: recommendations of the Nomenclature Committee on Cell Death 2012. Cell Death Differ. 2012;19(1):107-120.

25. Pelgrift RY, Friedman AJ. Nanotechnology as a therapeutic tool to combat microbial resistance. Adv Drug Deliv Rev. 2013;65(13-14): $1803-1815$.

26. Friberg S, Nyström AM. NANOMEDICINE: will it offer possibilities to overcome multiple drug resistance in cancer? J Nanobiotechnology. 2016;14:17.

27. Vinardell MP, Mitjans M. Antitumor activities of metal oxide nanoparticles. Nanomaterials. 2015;5(2):1004-1021.

28. Maiyo F, Singh M. Selenium nanoparticles: potential in cancer gene and drug delivery. Nanomedicine. 2017;12(9):1075-1089.

29. Woźniak A, Malankowska A, Nowaczyk G, et al. Size and shapedependent cytotoxicity profile of gold nanoparticles for biomedical applications. J Mater Sci Mater Med. 2017;28(6):92.

30. Deng J, Yao M, Gao C. Cytotoxicity of gold nanoparticles with different structures and surface-anchored chiral polymers. Acta Biomater. 2017;53:610-618.

31. Gaharwar US, Meena R, Rajamani P. Iron oxide nanoparticles induced cytotoxicity, oxidative stress and DNA damage in lymphocytes. $J$ Appl Toxicol. 2017;37(10):1232-1244.

32. Rene HD, Badireddy AR, José MS, et al. Cytotoxic effect of lipophilic bismuth dimercaptopropanol nanoparticles on epithelial cells. J Nanosci Nanotechnol. 2016;16(1):203-209.

33. Taylor DN, Hamer DH, Shlim DR. Medications for the prevention and treatment of travellers' diarrhea. J Travel Med.2017;24(suppl_1): S17-S22.

34. Hernandez-Delgadillo R, Badireddy AR, Zaragoza-Magaña V, SánchezNájera RI, Chellam S, Cabral-Romero C. Effect of lipophilic bismuth nanoparticles on erythrocytes. J Nanomater. 2015;2015(4):1-9.

35. Wang Y, Zi XY, Su J, et al. Cuprous oxide nanoparticles selectively induce apoptosis of tumor cells. Int J Nanomedicine. 2012;7: 2641-2652.

36. Chengzheng W, Jiazhi W, Shuangiiang C, et al. Biogenic synthesis, characterization and evaluation of silver nanoparticles from Aspergillus niger JX556221 against human colon cancer cell line HT-29. J Nanosci Nanotechnol. 2018;18(5):3673-3681.

37. Pérez-Herrero E, Fernández-Medarde A. Advanced targeted therapies in cancer: drug nanocarriers, the future of chemotherapy. Eur J Pharm Biopharm. 2015;93:52-79.

38. Li B, Zhang X, Dong Y. Nanoscale platforms for messenger RNA delivery. Wiley Interdiscip Rev Nanomed Nanobiotechnol. $2018 ; \mathrm{e} 1530$ 
39. Singh AK, Tiwari R, Kumar V. Photo-induced biosynthesis of silver nanoparticles from aqueous extract of Dunaliella salina and their anticancer potential. J Photochem Photobiol B. 2017;166:202-211.

40. Ahamed M, Akhtar MJ, Alhadlaq HA, Alshamsan A. Copper ferrite nanoparticle-induced cytotoxicity and oxidative stress in human breast cancer MCF-7 cells. Colloids Surf B Biointerfaces. 2016;142: 46-54.

41. Peters GJ, Adema AD, Bijnsdorp IV, Sandvold ML. Lipophilic prodrugs and formulations of conventional (deoxy)nucleoside and fluoropyrimidine analogs in cancer. Nucleosides Nucleotides Nucleic Acids. 2011; 30(12):1168-1180.

42. Wright BD, Deblock MC, Wagers PO, et al. Anti-tumor activity of lipophilic imidazolium salts on select NSCLC cell lines. Med Chem Res. 2015;24(7):2838-2861.
43. Lebedová J, Hedberg YS, Odnevall Wallinder I, Karlsson HL. Sizedependent genotoxicity of silver, gold and platinum nanoparticles studied using the mini-gel comet assay and micronucleus scoring with flow cytometry. Mutagenesis. 2018;33(1):77-85.

44. Liu YK, Deng XX, Yang HL. Cytotoxicity and genotoxicity in liver cells induced by cobalt nanoparticles and ions. Bone Joint Res. 2016; 5(10):461-469.

45. Banu H, Renuka N, Faheem SM, et al. Gold and silver nanoparticles biomimetically synthesized using date palm pollen extract-induce apoptosis and regulate p53 and Bcl-2 expression in human breast adenocarcinoma cells. Biol Trace Elem Res. 2018.

46. Azizi M, Ghourchian H, Yazdian F, Dashtestani F, Alizadehzeinabad H. Cytotoxic effect of albumin coated copper nanoparticle on human breast cancer cells of MDA-MB 231. PLoS One. 2017;12(11):e0188639.
International Journal of Nanomedicine

\section{Publish your work in this journal}

The International Journal of Nanomedicine is an international, peerreviewed journal focusing on the application of nanotechnology in diagnostics, therapeutics, and drug delivery systems throughout the biomedical field. This journal is indexed on PubMed Central, MedLine, CAS, SciSearch $®$, Current Contents $\AA /$ Clinical Medicine,

\section{Dovepress}

Journal Citation Reports/Science Edition, EMBase, Scopus and the Elsevier Bibliographic databases. The manuscript management system is completely online and includes a very quick and fair peer-review system, which is all easy to use. Visit http://www.dovepress.com/ testimonials.php to read real quotes from published authors.

Submit your manuscript here: http://www.dovepress.com/international-journal-of-nanomedicine-journal 